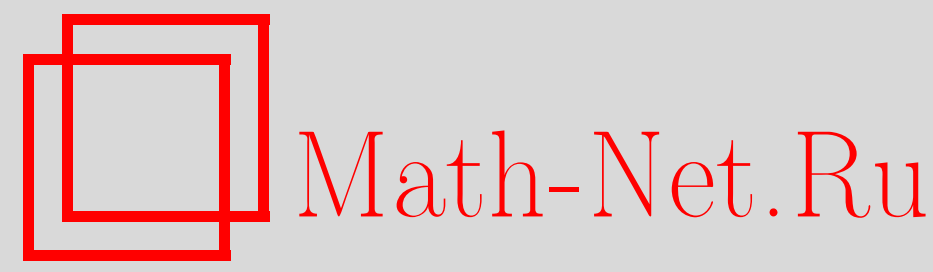

Ю. Г. Прохоров, О дополняемости канонического дивизора для расслоений Мори на коники, Матем. сб., 1997, том 188, номер 11, 99-120

DOI: https://doi.org/10.4213/sm277

Использование Общероссийского математического портала Math-Net.Ru подразумевает, что вы прочитали и согласны с пользовательским соглашением

http://www . mathnet.ru/rus/agreement

Параметры загрузки:

IP: 54.162 .27 .143

26 апреля 2023 г., $13: 29: 23$ 
УДК 512.7

\author{
Ю.Г. Прохоров
}

\title{
О дополняемости канонического дивизора для расслоений Мори на коники
}

\begin{abstract}
Настоящая статья продолжает изучение экстремалњных стягиваний с трехмерных многообразий на поверхность. Обсуждается гипотеза М. Рида о дополняемости канонического дивизора ("общем слоне") и гипотеза об особенностях базы. Такие стягивания естественно появляются в бирациональной классификации трехмерных алгебраических многообразий. Ситуация изучается локально вблизи вырожденного слоя.

Библиограф̆ия: 28 названий.
\end{abstract}

\section{Введение}

Мы продолжим изучение экстремальных стягиваний с трехмерных многообразий на поверхности (так называемых расслоений Мори на коники), начатое в [1]-[4].

ОПРЕДЕЛЕНИЕ 0.1. Пусть $(X, C)$ - росток трехмерного нормального комплексного пространства $X$ вдоль компактной приведенной кривой $C$ и пусть $(S, o)-$ росток нормального двумерного комплексного пространства $S$ в $o \in S$. Предположим, что $X$ имеет особенности не хуже чем терминальные. Собственный морфизм $f:(X, C) \rightarrow(S, o)$ называется расслоением Мори на коники, если

(i) $f^{-1}(o)_{\text {red }}=C$,

(ii) $f_{*} \mathscr{O}_{X}=\mathscr{O}_{S}$,

(iii) дивизор $-K_{X} f$-обилен.

Первым примером расслоений Мори на коники является расслоение на коники в классическом смысле: $f:(X, C) \rightarrow(S, o)$ называется (обычным) расслоением на коники, если $(S, o)$ - неособая точка и сушествует вложение $i:(X, C) \hookrightarrow \mathbb{P}^{2} \times(S, o)$ такое, что $\mathscr{O}_{\mathbb{P}^{2} \times S}(X)=\mathscr{O}_{\mathbb{P}^{2} \times S}(2,0)$.

Естественно возникающий в теории минимальных моделей вопрос - классификация расслоений Мори на коники. Следующие две гипотезы интересны для приложений программы Саркисова к изучению бирациональных свойств трехмерных многообразий со структурой расслоений на коники (см. [5], [6]).

ГиПоТЕЗА 0.2 (специальный случай гипотезы об обшем слоне М. Рида). Пусть $f:(X, C) \rightarrow(S, o)$ - расслоение Мори на коники. Тогда общий элемент антиканонической линейной системы $\left|-K_{X}\right|$ имеет лишь дювалевские особенносmu.

Работа вьполнена при частичной поддержке Российского фонда фундаментальных исследований (грант № 96-01-00820) и Международного научного фонда (грант № М9О3000). 
ГИПОТЕЗА 0.3. Пусть $f:(X, C) \rightarrow(S, o)$ - расслоение Мори на коники. Тогда точка $\left(S\right.$, о) неособа или является дювалевской особенностью типа $A_{n}$.

ЗАмечание 0.4. В работе [7] В.В. Шокуровым было введено понятие дополнения. В нашей ситуации это определение вьглядит следуюшим образом:

канонический дивизор $K_{X}$ называется 1-дополняемым, если для некоторого

$F \in\left|-K_{X}\right|$ логдивизор $K_{X}+F$ логканоничен.

Согласно $[7,3.2 .3]$ и обрашению присоединения $[7,3.3,9.5]$ общий дивизор $F \in\left|-K_{X}\right|$ имеет дювалевские особенности тогда и только тогда, когда $K_{X}+F$ чисто логтерминален (здесь используется то, что $K_{X}+F$ и $K_{F}-$ дивизоры индекса 1, поэтому все дискрепантности - целые). Таким образом, гипотеза 0.2 эквивалентна 1 -дополняемости $K_{X}$ в сильном смысле.

Вероятно следует ожидать, что расслоение Мори на коники с достаточно особой базой является фактором обычного расслоения на коники по циклической группе (например, это следует из гипотезы 0.2 , см. теорему 4.2). Таким образом, этот частньй случай, возможно, является довольно общим. Такие факторы классифицированы в $\S 2$. К счастью, ситуация здесь не очень сложна и с точностью до аналитического изоморфизма имеются лишш три различных расслоения Мори на коники. В $\S 3$ будут полностью описаны расслоения Мори на коники индекса 2. Предполагая истинность гипотезы 0.2 , мы получим грубую классификацию расслоений Мори на коники в $\S 4$. В частности, мы покажем (см. также [1]), что гипотеза 0.2 влечет гипотезу 0.3. Мы рассмотрим также много примеров. Используемые нами методы полностью элементарны. Здесь не используется техника работ Мори [8] или Шокурова [7]. Почти все результаты этой работы были анонсированы в препринтах [2] и [3].

Автор работал надэтой проблемой в институте Макса Планка в феврале-апреле 1995 и математическом институте университета Варвика в ноябре-декабре 1995. Автор выражает глубокую благодарность сотрудникам этих институтов за гостеприимство. Различные аспекты работы обсуждались с В. А. Исковских, В. В. Шокуровым, М. Ридом и Д. Орловым. Я благодарен им за помошь и советы.

\section{§ 1. Предварительные сведения}

Всюду в этой статье под многообразием мы понимаем приведенное неприводимое комплексное пространство. Мы будем обозначать канонический дивизор (Вейля) нормального многообразия $X$ через $K_{X}$. Если $X-$ многообразие и $C \subset X$ - его подмногообразие, то через $(X, C)$ будет обозначаться росток $X$ вдоль $C$. Иногда мы будем заменять $(X, C)$ на достаточно малый представитель $X \supset C$. Мы говорим, что многообразие $X$ имеет терминальные особенности, подразумевая, что оно имеет особенности не хуже чем терминальные, в частности, $X$ может быть неособым.

Нам понадобятся некоторые факты из классификации трехмерных терминальных особенностей.

Пусть $(X, P)$ - терминальная особенность индекса $m \geqslant 1$ и пусть $\pi:\left(X^{\#}, P^{\#}\right) \rightarrow$ $(X, P)$ - ее каноническое накрытие. Тогда $\left(X^{\#}, P^{\#}\right)$ - терминальная особенность 
индекса 1. Известно [9], что $\left(X^{\#}, P^{\#}\right)$ - гиперповерхностная особенность, т.е. существует $\mathbb{Z}_{m}$-эквивариантное вложение $\left(X^{\#}, P^{\#}\right) \subset\left(\mathbb{C}^{4}, 0\right)$.

Tеорема 1.1 [10]. Если в обозначениях выше точка $\left(X^{\#}, P^{\#}\right)$ неособа, то она $\mathbb{Z}_{m}$-изоморфна $\left(\mathbb{C}_{x_{1}, x_{2}, x_{3}}^{3}, 0\right)$ со следующим действием $\mathbb{Z}_{m}$

$$
\left(x_{1}, x_{2}, x_{3}\right) \longrightarrow\left(\varepsilon^{a} x_{1}, \varepsilon^{-a} x_{2}, \varepsilon^{b} x_{3}\right),
$$

әде $\varepsilon$ - первообразный корень степени $m$ из 1, и а, $b$ челье, взаимно простые с т иисла. Обратно, каждая такая особенность терминальна.

Такая особенность обычно обозначается $\frac{1}{m}(a,-a, b)$ или $\mathbb{C}^{3} / \mathbb{Z}_{m}(a,-a, b)$ и называется терминальной циклической факторособенностью.

Tеорема 1.2 [11], [12]. В обозначениях выше предположим, что точка $\left(X^{\#}, P^{\#}\right)$ особа, тогда она $\mathbb{Z}_{m}$-изоморфна гиперповерхности $\left\{\phi\left(x_{1}, x_{2}, x_{3}, x_{4}\right)\right.$ $=0\}$ в $\left(\mathbb{C}_{x_{1}, x_{2}, x_{3}, x_{4}}^{4}, 0\right)$, где имеются две возможсности для действия $\mathbb{Z}_{m}$ :

(i) (основная серия) $\left(x_{1}, x_{2}, x_{3}, x_{4} ; \phi\right) \longrightarrow\left(\varepsilon^{a} x_{1}, \varepsilon^{-a} x_{2}, \varepsilon^{b} x_{3}, x_{4} ; \phi\right)$, где $\varepsilon-$ первообразный корень степени т из 1, и $a, b$ - целые взаимно простые c $m$ числа,

(ii) (исключительная серия) $m=4 u$

$$
\left(x_{1}, x_{2}, x_{3}, x_{4} ; \phi\right) \rightarrow\left(\sqrt{-1} x_{1},-\sqrt{-1} x_{2}, \pm \sqrt{-1} x_{3},-x_{4} ;-\phi\right) .
$$

Циклические факторособенности обычно включаются в серию (i) с $\phi=x_{4}$.

ЗАмечАниЕ 1.3. Терминальные особенности индекса $>1$ могут быть классифицированы по типу общего элемента линейной системы $\left|-K_{X}\right|$ (который должен иметь дювалевскую особенность). Например, существуют четыре типа терминальных особенностей индекса 2: $c A / 2, c A x / 2, c D / 2$ и $c E / 2$ [12] (мы используем обозначения работы [13]).

Лемма 1.4. Пусть $(X, P)$ - росток трехмерной терминальной особенности и пусть $F \in\left|-K_{(X, P)}\right|$ - әлемент антиканонической линейной системь. Если $F$ - неприводимая неособая поверхность, то точка $(X, P)$ неособа.

ДокАЗАТЕЛЬство. По замечанию 0.4 логдивизор $K_{X}+F$ чисто логтерминален. Тогда точка $(X, P)$ неособа по $[7,3.7]$.

Хорошо известно, что любая дювалевская особенность $(F, P)$ (и более обшо, любая логтерминальная поверхностная особенность) является фактором неособого ростка $\left(\mathbb{C}^{2}, 0\right)$ по конечной группе $G$, действуюшей на $\mathbb{C}^{2}$ свободно вне 0 . Порядок $G$ называется топологическим индексом $(F, P)$. Аналогично лемме 1.4 можно доказать следуюшую лемму.

Лемма 1.5. Пусть $(X, P)$ - росток трехмерной терминальной особенности индекса $m>1$ и пусть $F \in \mid-K_{(X, P) \mid}$ - антиканонический дивизор. Предположим, что поверхность $F$ приведена, неприводима и точка $(F, P)$ дювалевская топологического индекса $n$. Тогда п делится на т. Более того, если $n=m$, то $(X, P)$ - циклическая факторособенность и $(F, P)$ имеет mun $A_{m-1}$. 
ДокАЗАТЕЛЬСТво. Как и выше, логдивизор $K_{X}+F$ чисто логтерминален. Пусть $\pi:\left(X^{\#}, P^{\#}\right) \rightarrow(X, P)$ - каноническое накрытие и $F^{\#}:=\pi^{-1}(F)$. Тогда $K_{X \#}+F^{\#}$ также чисто логтерминален $[7,2.2]$. Так как $F^{\# ~ с в я з е н ~}($ ср. $[7,5.7])$, то он неприводим и особенность $\left(F^{\#}, P^{\#}\right)$ - дювалевская. Накрытие $F^{\#} \rightarrow F$ является этальньм вне $P$, следовательно, топологический индекс $(F, P)$ делится на степень этого накрытия, равную $m$. Если достигается равенство $n=m$, то точка $\left(F^{\#}, P^{\#}\right)$ неособа и по лемме ??? вьше неособа также $\left(X^{\#}, P^{\#}\right)$.

Далее мы обсудим некоторые элементарные свойства расслоений Мори на коники.

ПРЕДЛОЖЕнИЕ 1.6 [14] (см. также 1.11). Пусть $f: X \rightarrow S$ - расслоение Мори на коники. Предположим, что $X$ содержит только точки индекса 1. Тогда поверхность $S$ неособа и $f$-расслоение на коники (возможно с особым $X$ ).

Следуюшее утверждение является немедленным следствием теоремы Каваматы-Фивега об обрашении в нуль.

ПРЕДЛОЖЕнИЕ 1.7 (ср. [8, (1.2)]). Пусть $f: X \rightarrow S$ - расслоение Мори на коники. Тогда

$$
R^{i} f_{*} \mathscr{O}_{X}=0, \quad i>0
$$

СлеДСТвИЕ 1.8 (ср. [8, (1.3)]). Пусть $f:(X, C) \rightarrow(S, o)$ - расслоение Мори на коники. Тогда

(i) слой $C$ - дерево рачиональньх кривых, т.е. $p_{a}\left(C_{0}\right) \leqslant 0$ для любой одномерной подсхеми $C_{0} c \operatorname{Supp}\left(C_{0}\right) \subset C$;

(ii) $\operatorname{Pic} X \simeq H^{2}(C, \mathbb{Z}) \simeq \mathbb{Z}^{\rho}$, әде $\rho$ - число компонент $C$.

1.9. Конструкция. Пусть $f:(X, C) \rightarrow(S, o)$ - расслоение Мори на коники. Предположим, что точка $(S, o)$ особа. По легкому замечанию в [15] (см. также [16] $),(S, o)$ - факторособенность. Это означает, что $(S, o)$ - фактор неособой точки $\left(S^{\prime}, o^{\prime}\right)$ по конечной группе $G$, где действие $G$ на $S^{\prime}-o^{\prime}$ свободно. Следовательно, сушествует точное представление $G \hookrightarrow \mathrm{GL}\left(T_{S^{\prime}, o^{\prime}}\right)=\mathrm{GL}_{2}(\mathbb{C})$. Обозначим через $g: S^{\prime} \rightarrow S$ факторморфизм и через $X^{\prime}$ нормализацию $X \times_{S} S^{\prime}$. Мы имеем следуюшую коммутативную диаграмму

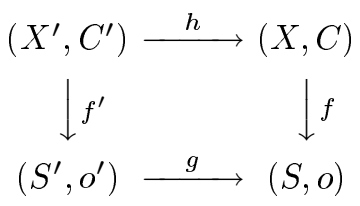

где $C^{\prime}:=f^{\prime-1}\left(o^{\prime}\right)$. Тогда $G$ действует на $\left(X^{\prime}, C^{\prime}\right)$ и, очевидно, $X=X^{\prime} / G$ и $C=$ $C^{\prime} / G$. Так как действие $G$ на $S^{\prime}-o^{\prime}$ свободно, свободно также действие на $X^{\prime}-C^{\prime}$. Поэтому $X^{\prime}$ имеет только терминальные особенности, так как $\operatorname{codim} C^{\prime}=2$ (см., например, $[17,6.7])$. Это дает нам, что действие $G$ на $X^{\prime}$ свободно вне конечного множества точек $Q_{1}, \ldots, Q_{k} \in X^{\prime}$ (для каждой точки $Q_{i}$ ее образ $P_{i}=h\left(Q_{i}\right)$ имеет индекс $>1)$. Так как $h: X^{\prime} \rightarrow X$ не имеет дивизоров ветвления, антиканонический дивизор $-K_{X^{\prime}}=h^{*}\left(-K_{X}\right)$ обилен над $S^{\prime}$. Мы получим новое расслоение Мори на коники $f^{\prime}:\left(X^{\prime}, C^{\prime}\right) \rightarrow\left(S^{\prime}, o^{\prime}\right)$ над неособой базой.

С. Мукай указал автору, что, на самом деле, $X \times{ }_{S} S^{\prime}$ нормально, следовательно, мы можем положить $X^{\prime}=X \times{ }_{S} S^{\prime}$. 
Лемма 1.10 (см. [1], [2], [18]). В обозначениях n. 1.9, группа $G$ является ииклической и имеет по крайней мере одну неподвижную точку на $X^{\prime}$. B частности, $(S$, о) - чиклическая факторособенность.

ДокАЗАТЕльСтво. Так как $p_{a}\left(C^{\prime}\right)=0$, то легко показать индукцией по числу компонент $C$, что $G$ имеет неподвижную точку $Q \in C^{\prime}$ или инвариантную компоненту $C_{0}^{\prime} \subset C^{\prime}, C_{0}^{\prime} \simeq \mathbb{P}^{1}$. Но во втором случае имеются два вложения $G \subset \mathrm{PGL}_{2}(\mathbb{C})$ и $G \subset \mathrm{GL}_{2}(\mathbb{C})$, где $G \subset \mathrm{GL}_{2}(\mathbb{C})$ не содержит квазиотражений. По классификации конечных подгрупп, а $\mathrm{PGL}_{2}(\mathbb{C}) \simeq \mathrm{SO}_{3}(\mathbb{C})$, группа $G$ является циклической и имеет ровно две неподвижных точки на $C_{0}^{\prime}$. Следовательно, в каждом случае $G$ имеет по крайней мере одну неподвижную точку $Q \in X^{\prime}$. Выберем достаточно малую окрестность $U \subset X$ точки $P:=h(Q)$. Имеется сюръективное отображение $\pi_{1}(U-P) \rightarrow G$. Но группа $\pi_{1}(U-P)$ - циклическая, так как $(X, P)-$ фактор гиперповерхностной особенности по циклической группе. Это доказьвает лемму.

СлЕДСТвИЕ 1.11. Пусть $f:(X, C) \rightarrow(S, o)-$ расслоение Мори на коники. Тогда

(i) (см. [14]) если X имеет индекс 1, то точка $(S$, о) неособа;

(ii) если $X$ имеет индекс 2 , то точка $(S, o)$ неособа или дювалевская muna $A_{1}$

(iii) если $(S$, o) имеет топологический индекс $n$, то $X$ содержит по крайней мере одну точку индекса $\geqslant n$.

\section{§ 2. Факторы расслоений на коники}

По п. 1.9 и лемме 1.10 каждое расслоение Мори на коники $f:(X, C) \rightarrow$ $(S, o)$ над особой базой является фактором другого расслоения Мори на коники $f^{\prime}:\left(X^{\prime}, C^{\prime}\right) \rightarrow\left(S^{\prime}, o^{\prime}\right)$ над неособой базой по циклической группе. В этом параграфе мы классифицируем расслоения Мори на коники $f:(X, C) \rightarrow(S, o)$ при дополнительном предположении, что многообразие $X^{\prime}$ горенштейново (и тогда $f^{\prime}:\left(X^{\prime}, C^{\prime}\right) \rightarrow\left(S^{\prime}, o^{\prime}\right)$ - обычное расслоение на коники по предложению 1.6). Сначала мы рассмотрим несколько примеров.

Пример 2.1 (торический пример). Пусть $\mathbb{P}^{1} \times \mathbb{C}^{2} \rightarrow \mathbb{C}^{2}$-проекция. Определим действие групшы $\mathbb{Z}_{n}$ на $\mathbb{C}_{u, v}^{2}$ и $\mathbb{P}_{x_{0}, x_{1}}^{1} \times \mathbb{C}_{u, v}^{2}$ следующим образом:

$$
\left(x_{0}, x_{1} ; u, v\right) \rightarrow\left(x_{0}, \varepsilon x_{1} ; \varepsilon^{a} u, \varepsilon^{-a} v\right)
$$

где $\varepsilon=\exp (2 \pi i / n), a \in \mathbb{N}$ и $(n, a)=1$. Обозначим $X=\left(\mathbb{P}^{1} \times \mathbb{C}^{2}\right) / \mathbb{Z}_{n}, S=\mathbb{C}^{2} / \mathbb{Z}_{n}$. Тогда проекция $f: X \rightarrow S$ является расслоением Мори на коники. Многообразие $X$ имеет на слое $f^{-1}(0)$ ровно две терминальные точки $P_{1}, P_{2}$, которые являются циклическими факторами типа $\frac{1}{n}(a,-a, \pm 1)$, поверхность $S$ имеет в 0 дювалевскую точку типа $A_{n-1}$. $\mathbb{C}_{u, v}^{2}:$

ПримеР 2.2. Рассмотрим следуюшую неособую гиперповерхность в $\mathbb{P}_{x_{0}, x_{1}, x_{2}}^{2} \times$

$$
X^{\prime}:=\left\{x_{0}^{2}+v x_{1}^{2}+u x_{2}^{2}=0\right\} .
$$


Пусть $n$ - нечетное целое, $n=2 q+1$, где $q \in \mathbb{N}$. Определим действие $\mathbb{Z}_{n}$ на $\mathbb{P}^{2} \times \mathbb{C}^{2}$ как

$$
\left(x_{0}, x_{1}, x_{2}, u, v\right) \rightarrow\left(x_{0}, \varepsilon^{-q} x_{1}, \varepsilon^{q} x_{2}, \varepsilon u, \varepsilon^{-1} v\right),
$$

где $\varepsilon=\exp (2 \pi i / n)$. Если $\psi(u, v)$ - инвариант, то $\mathbb{Z}_{n}$ естественно действует на $X^{\prime}$. Как и в примере $2.1, f: X^{\prime} / \mathbb{Z}_{n} \rightarrow \mathbb{C}^{2} / \mathbb{Z}_{n}$ - расслоение Мори на коники. Особое множество $X^{\prime} / \mathbb{Z}_{n}$ состоит из двух терминальных циклических факторособенностей индекса $n$. Точка $(S, o)$ - дювалевская типа $A_{n-1}$.

ПримеР 2.3. Пусть $X^{\prime}$ - гиперповерхность в $\mathbb{P}_{x_{0}, x_{1}, x_{2}}^{2} \times \mathbb{C}_{u, v}^{2}$, заданная уравнением

$$
x_{0}^{2}+x_{1}^{2}+x_{2}^{2} \phi(u, v)=0
$$

где $\phi(u, v)$ не имеет кратњых множителей и содержит только мономы четной степени. Обозначим через $f^{\prime}: X^{\prime} \rightarrow \mathbb{C}^{2}$ естественную проекцию. Тогда $X^{\prime}$ имеет только одну особую точку $P^{\prime}=\left(x_{0}=x_{1}=u=v=0\right)$ на $f^{\prime-1}(0)$. Определим действие $\mathbb{Z}_{2}$ на $X^{\prime}$ и $\mathbb{C}^{2}$ как

$$
\left(x_{0}, x_{1}, x_{2}, u, v\right) \rightarrow\left(-x_{0}, x_{1}, x_{2},-u,-v\right) .
$$

Пусть $X=X^{\prime} / \mathbb{Z}_{2}, S=\mathbb{C}^{2} / \mathbb{Z}_{2}$. Единственная неподвижная точка на $X^{\prime}$ - точка $P^{\prime}$. Она задает точку $P \in X$ индекса 2 , и многообразие $X$ не содержит других особых точек. Поверхность $S$ имеет дювалевскую особенность типа $A_{1}$ в 0 . Можно различить следующие случаи для $\phi(u, v)$ :

$(1) \operatorname{mult}_{(0,0)}(\phi)=2$, тогда точка $(X, P)$ терминальная типа $c A / 2$;

$(2) \operatorname{mult}_{(0,0)}(\phi) \geqslant 4$, тогда точка $(X, P)$ терминальная типа $c A x / 2$.

TeOPема 2.4. Пусть $f:(X, C) \rightarrow(S, o)$ - расслоение Мори на коники. Предположим, что $f$ является фактором расслоения на коники $f^{\prime}:\left(X^{\prime}, C^{\prime}\right) \rightarrow$ $\left(\mathbb{C}^{2}, 0\right)$ по $\mathbb{Z}_{n}$ таким, что действие $\mathbb{Z}_{n}$ на $\mathbb{C}^{2} \backslash\{0\}$ свободно. Тогда существует аналитический изоморфизм между $f:(X, C) \rightarrow(S, o)$ и одним из примеров 2.1, 2.2 или 2.3. В частности, $(S, o)$ - дювалевская особенность тиna $A_{n-1}$.

ДокАЗАТЕЛЬСтво. Мы зафикисируем образуюшую $s \in \mathbb{Z}_{n}$ и для $\mathbb{Z}_{n}$-полуинварианта $z$ определим его веc $\operatorname{wt}(z)$ как целое число, определенное по модулю $n$, такое, что

$$
\operatorname{wt}(z) \equiv a \quad \bmod n \Longleftrightarrow s(z)=\varepsilon^{a} z,
$$

где $\varepsilon$ - первообразный корень степени $n$ из 1 .

По лемме Картана [19] можно выбрать координаты $u, v$ на $\mathbb{C}^{2}$ так, что действие $\mathbb{Z}_{m}$ будет линейньм, и тогда можно считать, что $u \rightarrow \varepsilon^{a} u, v \rightarrow \varepsilon^{b} v$, где $\varepsilon:=$ $\exp (2 \pi i / m)$, и $(a, m)=(b, m)=1$.

Пусть $X_{0}^{\prime}:=f^{\prime-1}(0)$ - схемно-теоретический слой над 0. Тогда $X_{0}^{\prime}$ изоморфен конике в $\mathbb{P}^{2}$ (см. предложение 1.6). Имеются следующие три случая $2.5,2.6$ и 2.9 . 
2.5. Слой $X_{0}^{\prime}-$ невырожденная коника. Тогда в аналитической ситуации по теореме Грауэрта-Фишера $X^{\prime} \simeq \mathbb{P}^{1} \times \mathbb{C}^{2}$ и мы можем предположить, что действие $\mathbb{Z}_{n}$ в некоторой системе координат $\left(x_{0}, x_{1}\right)$ в $\mathbb{P}^{1}$ и $(u, v)$ в $\mathbb{C}^{2}$ будет

$$
\left(x_{0}, x_{1}, u, v\right) \rightarrow\left(x_{0}, \varepsilon x_{1}, \varepsilon^{a} u, \varepsilon^{b} v\right), \quad \text { где }(a, m)=(b, m)=1
$$

(здесь мы можем положить wt $\left(x_{0}\right)=0$, так как $\left(x_{0}, x_{1}\right)$ - однородные координаты). Имеется ровно две неподвижные точки на $X^{\prime}$ :

$$
Q_{0}=\left\{x_{0}=u=v=0\right\}, \quad Q_{1}=\left\{x_{1}=u=v=0\right\} .
$$

Они задают нам две точки индекса $n$ на $X^{\prime} / \mathbb{Z}_{n}$ типов $\frac{1}{n}(-1, a, b)$ и $\frac{1}{n}(1, a, b)$ соответственно. По теореме 1.1 эти две точки терминальны, только если $a+b=n$ (напомним, что числа $a, b$ определены по модулю $n$ ). Мы получаем пример 2.1.

2.6. Слой $X_{0}^{\prime}$ приводим. Тогда $X_{0}^{\prime}=L_{1}+L_{2}$ - пара прямых, пересекающихся в одной точке, которая является неподвижной относительно действия $\mathbb{Z}_{n}$. Обозначим эту точку через $Q$. Пусть $P:=h(Q)$. Аналогично случаю 2.5 рассмотрим $\mathbb{Z}_{n}$-эквивариантное вложение $X^{\prime} \subset \mathbb{P}_{x_{0}, x_{1}, x_{2}}^{2} \times \mathbb{C}_{u, v}^{2}$ такое, что координаты $x_{0}, x_{1}, x_{2}$ - полуинварианты с весами

$$
\operatorname{wt}\left(x_{0}, x_{1}, x_{2} ; u, v\right)=(0, p, q ; a, b), \quad(a, n)=(b, n)=1 .
$$

Имеются два подслучая 2.7 и 2.8 .

2.7. Компоненты $X_{0}^{\prime}$ являются $\mathbb{Z}_{n}$-инвариантными. Покажем, что это невозможно. Мы можем выбрать однородные координаты $x_{0}, x_{1}, x_{2}$ в $\mathbb{P}^{2}$ так, что уравнение $X_{0}^{\prime} \subset \mathbb{P}^{2}$ будет $\left\{x_{0} x_{1}=0\right\}$. Тогда $X^{\prime}$ задается уравнением

$$
\begin{aligned}
x_{0} x_{1}+\varphi_{0}(u, v) x_{0}^{2}+\varphi_{1}(u, v) x_{0} x_{1} & +\varphi_{2}(u, v) x_{0} x_{2} \\
& +\phi(u, v) x_{1}^{2}+\psi(u, v) x_{1} x_{2}+\zeta(u, v) x_{2}^{2}=0
\end{aligned}
$$

где $\varphi_{0}(0,0)=\varphi_{1}(0,0)=\varphi_{2}(0,0)=\phi(0,0)=\psi(0,0)=\zeta(0,0)=0$. Сделав замену $x_{1}^{\prime}=x_{1}+\varphi_{0} x_{0}+\varphi_{1} x_{1}+\varphi_{2} x_{2}$, мы получим новое уравнение для $X^{\prime}$ :

$$
x_{0} x_{1}+\phi(u, v) x_{1}^{2}+\psi(u, v) x_{1} x_{2}+\zeta(u, v) x_{2}^{2}=0
$$

где $\phi(u, v), \psi(u, v), \zeta(u, v)$ - полуинварианты с подходящими весами и такие, что $\phi(0,0)=\psi(0,0)=\zeta(0,0)=0$. Тогда $Q=\left\{x_{0}=x_{1}=u=v=0\right\}$ и $\left(y_{0}:=\right.$ $\left.x_{0} / x_{2}, y_{1}:=x_{1} / x_{2}, u, v\right)$ - локальные координаты в $\mathbb{P}^{2} \times \mathbb{C}^{2}$ в окрестности $Q$. Легко видеть, что

$$
(X, P) \simeq\left\{y_{0} y_{1}+\phi(u, v) y_{1}^{2}+\psi(u, v) y_{1}+\zeta(u, v)=0\right\} / \mathbb{Z}_{n}(-q, p-q, a, b)
$$

Действие $\mathbb{Z}_{n}$ на $X^{\prime}$ имеет еще две неподвижные точки $Q_{1}:=\left\{x_{0}=x_{2}=u=v=0\right\}$ и $Q_{2}:=\left\{x_{1}=x_{2}=u=v=0\right\}$. Тогда $\left(z_{0}=x_{0} / x_{1}, z_{2}=x_{2} / x_{1}, u, v\right)$-локальные координаты в $\mathbb{P}^{2} \times \mathbb{C}^{2}$ в окрестности $Q_{1}$ и, аналогично, $\left(t_{1}=x_{1} / x_{0}, t_{2}=x_{2} / x_{0}, u, v\right)$ 
- локальные координаты в $\mathbb{P}^{2} \times \mathbb{C}^{2}$ в окрестности $Q_{2}$. Положим $P_{i}:=h\left(Q_{i}\right)$, $i=1,2$. Аналогично (2) имеем

$$
\left(X, P_{1}\right) \simeq\left\{z_{0}+\phi(u, v)+\psi(u, v) z_{2}+\zeta(u, v) z_{2}^{2}=0\right\} / \mathbb{Z}_{n}(-p, q-p, a, b)
$$

и

$$
\left(X, P_{2}\right) \simeq\left\{t_{1}+\phi(u, v) t_{1}^{2}+\psi(u, v) t_{1} t_{2}+\zeta(u, v) t_{2}^{2}=0\right\} / \mathbb{Z}_{n}(p, q, a, b) .
$$

Отсюда

$$
\begin{aligned}
& \left(X, P_{1}\right) \simeq \mathbb{C}_{z_{2}, u, v}^{3} / \mathbb{Z}_{n}(q-p, a, b), \\
& \left(X, P_{2}\right) \simeq \mathbb{C}_{t_{2}, u, v}^{3} / \mathbb{Z}_{n}(q, a, b) .
\end{aligned}
$$

Так как действие $\mathbb{Z}_{n}$ на $X^{\prime}$ имеет лишь нульмерное множество неподвижных точек, то

$$
(q, n)=(p-q, n)=1 .
$$

Тогда по (2) и теореме $1.2(X, P)$ - циклическая факторособенность. Это возможно, только если $\zeta(u, v)$ содержит член $u$ или $v$. С точностью до перестановки $u, v$ мы можем предположить, что $\zeta=u+\cdots$. Из (1) получаем $\operatorname{wt}\left(x_{0} x_{1}\right)=\operatorname{wt}\left(u x_{2}^{2}\right)$. Таким образом, $p=a+2 q$. Тогда

$$
(X, P) \simeq \mathbb{C}_{y_{0}, y_{1}, v}^{3} / \mathbb{Z}_{n}(-q, p-q, b)=\mathbb{C}^{3} / \mathbb{Z}_{n}(-q, a+q, b) .
$$

Мы утверждаем, что $a+b=0$. Действительно, в противном случае $n>2$ и из (3) мы имеем $b=a+q$, так как $p=a+2 q$ и $(q, n)=1$. Следовательно, $(X, P) \simeq \mathbb{C}^{3} / \mathbb{Z}_{n}(-q, b, b)$. Эта точка не может быть терминальной, если $n>2$.

Противоречие показывает, что $a+b=0$. Точка $(X, P)$ терминальна в этом случае, только если $q=b$ (см. (5)). Но тогда $p-q=a+2 q-q=0$, противоречие c (5).

2.8. $\mathbb{Z}_{n}$ переставляет компоненты $X_{0}^{\prime}$. Тогда $n$ четно, $n=2 k$. Если $k>1$, то фактор $X^{\prime} / \mathbb{Z}_{k} \rightarrow \mathbb{C}^{2} / \mathbb{Z}_{k}$ является расслоением Мори на коники, таким как в п. 2.7. Мы уже доказали, что это невозможно. Следовательно, $k=1, n=2$. Тогда легко видеть, что $\operatorname{wt}(u)=\operatorname{wt}(v)=1$. Изменяя координаты, мы можем добиться того, что слой $X_{0}^{\prime} \subset \mathbb{P}^{2}$ задается как $\left\{x_{0}^{2}+x_{1}^{2}=0\right\}$. Так как $\mathbb{Z}_{2}$ переставляет компоненты $\left\{x_{0}^{2}+x_{1}^{2}=0\right\}$, мы можем предположить также, что $\mathbb{Z}_{2}$ действует на $x_{0}, x_{1}$ следуюшим образом: $x_{0} \rightarrow-x_{0}, x_{1} \rightarrow x_{1}$. Тогда можно изменить систему координат $\left(x_{0}, x_{1}, x_{2} ; u, v\right)$ в $\mathbb{P}^{2} \times \mathbb{C}^{2}$ так, что многообразие $X^{\prime}$ задается уравнением

$$
x_{0}^{2}+x_{1}^{2}+\phi(u, v) x_{2}^{2}=0,
$$

где $\phi(u, v)$ - инвариант с $\phi(0,0)=0$. Это означает, что $\phi$ содержит только мономы четной степени. Тогда $Q=\left\{x_{0}=x_{1}=u=v=0\right\}$ - единственная особая точка на $C^{\prime}$ и $y_{0}:=x_{0} / x_{2}, y_{1}:=x_{1} / x_{2}, u, v$ - локальные координаты на $\mathbb{P}^{2} \times \mathbb{C}^{2}$ в окрестности $Q$. Следовательно,

$$
(X, P) \simeq\left\{y_{0}^{2}+y_{1}^{2}+\phi(u, v)=0\right\} / \mathbb{Z}_{2} .
$$

Если эта точка терминальна, то с точностью до перестановки $y_{0}, y_{1}$ мы можем предположить, что $\operatorname{wt}\left(y_{0}\right)=1, \operatorname{wt}\left(y_{1}\right)=0$. Таким образом, $\operatorname{wt}\left(x_{2}\right)=0$. Наконец, особенности многообразия $X^{\prime}$ изолированы, только если $\phi$ не имеет кратных множителей. Таким образом, мы получаем пример 2.3 . 
2.9. Слой $X_{0}^{\prime}$ является двойной прямой. Как и выше, рассмотрим $\mathbb{Z}_{n}$-эквивариантное вложение $X^{\prime} \subset \mathbb{P}_{x_{0}, x_{1}, x_{2}}^{2} \times \mathbb{C}_{u, v}^{2}$ такое, что действие $\mathbb{Z}_{n}$ будет

$$
\operatorname{wt}\left(x_{0}, x_{1}, x_{2} ; u, v\right)=(0, p, q ; a, b), \quad(a, n)=(b, n)=1 .
$$

Мы можем предположить также, что слой $X_{0}^{\prime} \subset \mathbb{P}^{2}$ задается уравнением $\left\{x_{0}^{2}=0\right\}$. Тогда в некоторой системе координат $\left(x_{0}, x_{1}, x_{2} ; u, v\right)$ в $\mathbb{P}^{2} \times \mathbb{C}^{2}$, состоящей из полуинвариантов, многообразие $X$ задается уравнением

$$
x_{0}^{2}+\phi(u, v) x_{1}^{2}+\psi(u, v) x_{1} x_{2}+\zeta(u, v) x_{2}^{2}=0,
$$

где $\phi(u, v), \psi(u, v), \zeta(u, v)$ - полуинварианты такие, что $\phi(0,0)=\psi(0,0)=$ $\zeta(0,0)=0$. Локальной координатой вдоль слоя $X_{0}^{\prime}$ является $x_{1} / x_{2}$ и $\operatorname{wt}\left(x_{1} / x_{2}\right)=p-q$. Так как действие $\mathbb{Z}_{n}$ на $X^{\prime}$ свободно в коразмерности два, $(p-q, n)=1$. Изменяя образуюший группы $\mathbb{Z}_{n}$ мы можем добиться того, что $p-q=1$. Неподвижными точками являются $Q_{1}=\left\{u=v=0, x_{0}=x_{1}=0\right\}$ и $Q_{2}=\left\{u=v=0, x_{0}=x_{2}=0\right\}$. За локальные координаты вблизи $Q_{1}$ и $Q_{2}$ в $\mathbb{P}^{2} \times \mathbb{C}^{2}$ мы можем взять $\left(y_{0}=x_{0} / x_{2}, y_{1}=x_{1} / x_{2}, u, v\right)$ и $\left(z_{0}=x_{0} / x_{1}, z_{2}=x_{2} / x_{1}, u, v\right)$ соответственно. Точка $Q_{1} \in X^{\prime}$ задает особую точку $P_{1} \in X$ типа

$$
\left\{y_{0}^{2}+\phi(u, v) y_{1}^{2}+\psi(u, v) y_{1}+\zeta(u, v)=0\right\} / \mathbb{Z}_{n}(-q, 1, a, b) .
$$

Аналогично, точка $Q_{2} \in X^{\prime}$ задает особую точку $P_{2} \in X$ типа

$$
\left\{z_{0}^{2}+\phi(u, v)+\psi(u, v) z_{2}+\zeta(u, v) z_{2}^{2}=0\right\} / \mathbb{Z}_{n}(-p,-1, a, b) .
$$

Мы утверждаем, что точки $\left(X, P_{1}\right)$ и $\left(X, P_{2}\right)$ - из основной серии $1.2(\mathrm{i})$. Действительно, предположим, например, что $\left(X, P_{1}\right)$ - точка из исключительной серии 1.2 (ii). Тогда $n=4, q=2, p=3$. Для $P_{2}$ все веса взаимно просты с $n=4$. По теореме $1.2 P_{2}$ - циклическая факторособенность и многообразие $X^{\prime}$ неособо в $Q_{2}$. Это возможно, только если $\phi(u, v)$ содержит линейный член. Тогда $\operatorname{wt}(\phi)=\operatorname{wt}\left(z_{0}^{2}\right)=2$. Это дает нам $a=\operatorname{wt}(u)=2$ или $b=\operatorname{wt}(v)=2$, противоречие $\mathrm{c}(a, n)=(b, n)=1$.

Теперь мы утверждаем, что многообразие $X^{\prime}$ неособо в $Q_{1}$ и $Q_{2}$. Как выше, если $Q_{1} \in X^{\prime}$ - особая точка, то по теореме 1.2 имеем $q=0, p=1$. Снова по 1.2 $P_{2}$ - циклическая факторособенность и $X^{\prime}$ неособо в $Q_{2}$. Это возможно, только если $\phi(u, v)$ содержит линейный член. С точностью до перестановки $u, v$ мы можем считать, что $\phi=u+\cdots$. Так как $\operatorname{wt}(\phi)=\operatorname{wt}\left(z_{0}^{2}\right)=-2$, мы имеем $a=\operatorname{wt}(u)=-2$. Следовательно, $n$ нечетно и

$$
\begin{aligned}
& \left(X, P_{1}\right) \simeq\left\{y_{0}^{2}+\phi(u, v) y_{1}^{2}+\psi(u, v) y_{1}+\zeta(u, v)=0\right\} / \mathbb{Z}_{n}(0,1,-2, b) \\
& \left(X, P_{2}\right) \simeq\left\{z_{0}^{2}+(u+\cdots)+\psi(u, v) z_{2}+\zeta(u, v) z_{2}^{2}=0\right\} / \mathbb{Z}_{n}(-1,-1,-2, b) .
\end{aligned}
$$

Отсюда

$$
\left(X, P_{2}\right) \simeq \mathbb{C}_{z_{0}, z_{2}, v}^{3} / \mathbb{Z}_{n}(-1,-1, b) .
$$

Так как $P_{2}$ - терминальная точка и $n$ нечетно, из (9) имеем $b=1$. Но, с другой стороны, по (8) $1+b=0$ или $-2+b=0$, противоречие. 
Следовательно, многообразие $X^{\prime}$ неособо в $Q_{1}$ и $Q_{2}$. Из (6) и (7) следует, что $\phi(u, v)$ и $\zeta(u, v)$ содержат линейные члены. С точностью до перестановки $u, v$ мы можем считать, что $\zeta=u+\cdots$. Поэтому $a=-2 q$. Более того $\left(X, P_{1}\right)=$ $\frac{1}{n}(-q, 1, b)$. Если $\phi=u+\ldots$, то по $(7)-2 p=a=-2+a$, следовательно, $n=2$, $a=0$, противоречие. Таким образом, $\phi=v+\cdots$. Это дает нам $-2 p=b=a-2$ и $\left(X, P_{2}\right)=\frac{1}{n}(-p,-1, a)$. Так как $b$ четно, $n$ нечетно. Далее, $a=-2 q, p=q+1$ и $b=-2 q-2$. Таким образом,

$$
\begin{aligned}
& \left(X, P_{1}\right)=\frac{1}{n}(-q, 1,-2 q-2), \\
& \left(X, P_{2}\right)=\frac{1}{n}(-q-1,-1,-2 q) .
\end{aligned}
$$

Сейчас мы покажем, что $n=2 q+1$. Действительно, предположим противное. Тогда, так как точка $\left(X, P_{2}\right)$ терминальна, из $(10)$ мы имеем $q+2=0$ или $3 q+1=0$. Но в первом случае $\left(X, P_{1}\right)=\frac{1}{n}(2,1,2)$. Эта точка может быть терминальной, только если $n=3$ и $q=1$. Во втором случае точка $\left(X, P_{1}\right)=\frac{1}{n}(-q, 1,-2 q-2)$ не может быть терминальной. Таким образом, наше утверждение доказано. В частности, мы имеем $a=-2 q=1, b=-2 q-2=-1$. Наконец, в нашем случае $\operatorname{wt}\left(x_{0}, x_{1}, x_{2}, u, v\right)=(0,-q, q ; 1,-1)$ и, изменяя координаты в $\mathbb{C}^{2}$ следующим образом

$$
u^{\prime}=\zeta(u, v), \quad v^{\prime}=\phi(u, v),
$$

мы получим

$$
X \simeq\left\{x_{0}^{2}+v x_{1}^{2}+\psi(u, v) x_{1} x_{2}+u x_{2}^{2}=0\right\} / \mathbb{Z}_{2 q+1}(0,-q, q ; 1,-1) .
$$

Заменой координат, как в $[20,1.5 .2]$, можно исключить член $\psi(u, v) x_{1} x_{2}$. Следовательно, $X$ - такое, как в примере 2.2. Это доказывает теорему.

\section{§3. Расслоения Мори на коники индекса 2}

В этом параграфе мы изучим расслоения Мори на коники $f:(X, C) \rightarrow(S, o)$ индекса 2. Сначала мы рассмотрим случай неособой базы (т.е. $(S, o) \simeq\left(\mathbb{C}^{2}, 0\right)$ ). Методы, используемые здесь, аналогичны методам [13, ch. 4]. Первый основной результат настоящего параграфа - следуюшая

Teорема 3.1. Пусть $f:(X, C) \rightarrow(S, o)$ - расслоение Мори на коники над неособой поверхностью $(S, o) \simeq\left(\mathbb{C}^{2}, 0\right)$. Предположим что многообразие $X$ имеет индекс 2. Тогда

(i) $X$ содержит ровно одну точку $P$ индекса 2 , центральный слой $C$ имеет не более четырех компонент, все они проходят через $P$ и не пересекаются вне $P$.

(ii) $f:(X, C) \rightarrow(S$, o) является фактором әллиптического расслоения $g:(Y, L) \rightarrow(S, o)$ по группе $\mathbb{Z}_{2}$, действующей тривиально на $(S, o)$. Здесь $(Y, L)$ - росток трехмерного многообразия с горенштейновыми терминальными особенностями вдоль приведенной компактной кривой $L, L=\left(g^{-1}(o)\right)_{\mathrm{red}}$, и общий слой $Y_{s}:=g^{-1}(s), s \in S$, - неособая әллиптическая кривая. Более 
того, $K_{Y}$ (линейно) тривиален вблизи L. Таким образом, морфизм g разлагается в композииию

$$
g:(Y, L) \stackrel{h}{\longrightarrow}(X, C) \stackrel{f}{\longrightarrow}(S, o)
$$

әде $h$ - конечный морфизм степени 2.

(iii) Множество $\mathbb{Z}_{2}$-неподвижных точек на $Y$ - это изолированная точка $Q$ такая, что $h(Q)=P$, и неособая поверхность $D \not \supset Q$, лежсащая в неособой части $Y$.

(iv) Cуществует $\mathbb{Z}_{2}$-әквивариантное вложсение $Y \hookrightarrow \mathbb{P}^{3} \times \mathbb{C}^{2}$ такое, что $g$ совпадает с проекцией на второй множстель $\mathbb{C}^{2}=S$, а $Y$ является пересечением двух гиперповерхностей, имеющих степень 2 по первому множителю. При этом вложении действие $\mathbb{Z}_{2}$ на $\mathbb{P}_{x_{0}, x_{1}, x_{2}, x_{3}}^{3} \times \mathbb{C}_{u, v}^{2}$ может бъть записано в виде

$$
\left(x_{0}, x_{1}, x_{2}, x_{3} ; u, v\right) \longrightarrow\left(-x_{0},-x_{1},-x_{2}, x_{3} ; u, v\right)
$$

$u \operatorname{moгдa~} Q=\left\{x_{0}=x_{1}=x_{2}=u=v=0\right\}, D=\left\{x_{3}=0\right\} \cap Y$.

(v) При вложсении из (iv) кривая $L$ будет обгединением $\leqslant 4$ прямых, проходящих через $Q$. В частности, действие $\mathbb{Z}_{2}$ на $L$ не переставляет компонент.

(vi) $В$ условиях (iv) уравнения для $Y \subset \mathbb{P}_{x_{0}, x_{1}, x_{2}, x_{3}}^{3} \times \mathbb{C}_{u, v}^{2}$ могут бить записаны в виде

$$
\left\{\begin{array}{l}
q_{1}\left(x_{0}, x_{1}, x_{2}\right)=\psi_{1}\left(x_{0}, x_{1}, x_{2}, x_{3} ; u, v\right) \\
q_{2}\left(x_{0}, x_{1}, x_{2}\right)=\psi_{2}\left(x_{0}, x_{1}, x_{2}, x_{3} ; u, v\right)
\end{array}\right.
$$

где $\psi_{1}, \psi_{2}$ - инвариантьл, квадратичные по $x_{0}, x_{1}, x_{2}, x_{3}$ и $\psi_{i}(0,0,0,1 ; 0,0)=0$, $i=1,2$, а для $q_{1}, q_{2}$ имеются следующие возможсности:

(a) $q_{1}=x_{0} x_{1}, q_{2}=\left(x_{0}+x_{1}+x_{2}\right) x_{2}$, тогда иентральный слой $f^{-1}($ о) имеет ровно четыре приведенные компоненты,

(b) $q_{1}=x_{0} x_{1}, q_{2}=\left(x_{0}+x_{1}\right) x_{2}$, тогда иентральный слой $f^{-1}($ о) имеет три компонентьи, одна из них имеет кратность 2,

(c) $q_{1}=x_{0} x_{1}-x_{2}^{2}, q_{2}=x_{0} x_{2}$, тогда центральный слой $f^{-1}($ о) имеет две компоненты, одна из них имеет кратность 3 ,

(d) $q_{1}=x_{0} x_{1}, q_{2}=x_{2}^{2}$, тогда чентральный слой $f^{-1}($ о) имеет две компонентыл, обе двукратные,

(е) $q_{1}=x_{0} x_{1}-x_{2}^{2}, q_{2}=x_{0}^{2}$, тогда иентральньй слой $f^{-1}($ о) неприводим и имеет кратность 4 ,

(f) $q_{1}=x_{0}^{2}, q_{2}=x_{1}^{2}$, тогда центральный слой $f^{-1}($ о $)$ также неприводим и имеет кратность 4 .

ЗАМЕЧАНИЕ 3.2. Обратно, пусть $g:(Y, L) \rightarrow(S, o) \simeq\left(\mathbb{C}^{2}, 0\right)$ - эллиптическое расслоение с действием $\mathbb{Z}_{2}$, такое как в (i)-(ii) теоремы 3.1. Если точка $(Y, Q) / \mathbb{Z}_{2}$ терминальна, то $f:(X, C):=(Y, L) / \mathbb{Z}_{2} \rightarrow(S, o)-$ расслоение Мори на коники индекса два.

ДокАЗАТЕЛьство. Мы докажем теорему 3.1 в несколько этапов. 
ЛЕмма 3.3. Пусть $f: X \rightarrow S$-морфизм из нормального трехмерного многообразия с только терминальныци особенностями на неособую поверхность. Предположим, что все слои $f$ связны и одномерны. Тогда морфизм $f: X \rightarrow S$ плоский.

ДоказАТЕЛьство. Терминальные особенности рациональны $[21,1-3-6]$ и, следовательно, $X$ - многообразие Коэна-Маколея [22]. Тогда $f$ плоский по [23, 23.1].

3.4. Конструкция (cp. $[13, \mathrm{ch} .4])$. Положим $m_{i}:=\left(-2 K_{X} \cdot C_{i}\right)$. Пусть $B_{i}$ - объединение $m_{i}$ различных дисков, пересекающих $C_{i}$ трансверсально в общих точках, и пусть $B=\sum B_{i}$. Так как $\operatorname{Pic} X=\mathbb{Z}^{\rho}$, то $B \in\left|-2 K_{X}\right|$. Рассмотрим двулистное накрытие $h: Y \rightarrow X$ с дивизором ветвления $B$. Пусть также $L:=$ $\left(h^{-1}(C)\right)_{\text {red }}$ и $D:=\left(h^{-1}(B)\right)_{\text {red }}$. Мы имеем композицию

$$
g:(Y, L) \stackrel{h}{\longrightarrow}(X, C) \stackrel{f}{\longrightarrow}(S, o) \simeq\left(\mathbb{C}^{2}, 0\right) .
$$

Тогда $(X, C) \simeq(Y, L) / \mathbb{Z}_{2}$. По построению $Y$ нормально, поэтому слои $g: Y \rightarrow \mathbb{C}^{2}$ связны. В окрестности каждой особой точки на $X$ морфизм $h$ неразветвлен в коразмерности 1 , следовательно, $Y$ имеет лишш терминальные особенности (см, например, $[9,(3.1)]$ или $[17,(6.7)])$. Далее, мы имеем следующие равенства для дивизоров Вейля на $Y$

$$
K_{Y}=h^{*}\left(K_{X}\right)+D, \quad D=h^{*}\left(-K_{X}\right) .
$$

Это дает нам $K_{Y}=0$ и то, что $Y$ имеет лишь терминальные горенштейновы особенности. Морфизм $g$ плоский по лемме 3.3. Следовательно, $g$ - расслоение на эллиптические кривые. Мы доказали (ii) и (iii).

3.5. Пусть $Y_{s}:=g^{-1}(s)$ - схемно-теоретический слой над точкой $s \in S$ (таким образом, $\left.L=\left(Y_{o}\right)_{\text {red }}\right)$. Тогда $Y_{s}$ - проективная горенштейнова одномерная схема и по формуле присоединения $\omega_{Y_{s}}=\mathscr{O}_{Y_{s}}$, в частности, $p_{a}\left(Y_{s}\right)=1$. Рассмотрим дивизор Картье $A:=\left.D\right|_{Y_{s}}$. Так как морфизм $g$ плоский, то $\operatorname{deg} A=4$.

ПРЕДЛОЖЕНИЕ 3.6. Пусть $Y_{s}$ и $A$ - такие как в n. 3.5. Тогда

(i) для любой замкнутой одномерной подсхемь $Z \subset Y_{s}, Z \neq Y_{s}$, имеем $p_{a}(Z) \leqslant 0$

(ii) дивизор А очень обилен и определяет вложение $Y_{s} \hookrightarrow \mathbb{P}^{3}$ такое, ито $Y_{s}$ является пересечением двух квадрик.

ДокАЗАТЕЛЬСтво. Докажем сначала импликацию (i) $\Rightarrow$ (ii). Мы воспользуемся следуюшим обобшенным вариантом известного классического результата.

Теорема 3.7 [24]. Пусть $Z$ - проективная одномерная схема Коэна-Маколея и $A$-дивизор Картье на $Z$. Предположим, что для любой замкнутой одномерной подсхемы $Z^{\prime} \subset Z$, горенштейновой в коразмерности 1 , имеет место неравенство

$$
\left.\operatorname{deg} A\right|_{Z^{\prime}} \geqslant 2 p_{a}\left(Z^{\prime}\right)+1
$$

Тогда А очень обилен.

Используя теорему Римана-Роха на $Y_{s}$, двойственность Серра и обильность $A$ получим $h^{1}\left(\mathscr{O}_{Y_{s}}(A)\right)=0, h^{0}\left(\mathscr{O}_{Y_{s}}(A)\right)=4$. Применим теорему 3.7 к $Y_{s}$. Если 
$Z^{\prime}=Y_{s}$, то $p_{a}\left(Z^{\prime}\right)=1$ и неравенство (12), очевидно, верно. Если же $Z^{\prime} \neq Y_{s}$, то неравенство (12) верно по предложению 3.6 (i). Далее из точной последовательности

$$
0 \longrightarrow \mathscr{I}(2) \longrightarrow \mathscr{O}_{\mathbb{P}^{3}}(2) \longrightarrow \mathscr{O}_{Y_{s}}(2 A) \longrightarrow 0,
$$

где $\mathscr{I}$ - пучок идеалов $Y_{s}$ в $\mathbb{P}^{3}$, нетрудно получить, что $h^{0}(\mathscr{I}(2)) \geqslant 2$. Так как $\operatorname{deg} Y_{s}=4$, то две квадрики $q_{1}, q_{2} \in H^{0}\left(\mathbb{P}^{3}, \mathscr{I}(2)\right)$ высекают $Y_{s}$. Это доказывает (ii).

Теперь докажем (i). Имеем сюръекцию $\left(R^{1} g_{*} \mathscr{O}_{Y}\right)_{s} \rightarrow H^{1}\left(Y_{s}, \mathscr{O}_{Y_{s}}\right) \rightarrow 0$. Так как $\omega_{Y / S}=\mathscr{O}_{Y}$, то пучок $R^{1} g_{*} \mathscr{O}_{Y}$ локально свободен по [25]. Следовательно, $s \rightarrow h^{1}\left(Y_{s}, \mathscr{O}_{Y_{s}}\right)$ - постоянная функция. По двойственности Серра $h^{0}\left(Y_{s}, \mathscr{O}_{Y_{s}}\right)=$ $h^{1}\left(Y_{s}, \mathscr{O}_{Y_{s}}\right)=1$ для всех $s \in S$. Рассмотрим точную последовательность

$$
0 \longrightarrow \mathscr{J} \longrightarrow \mathscr{O}_{Y_{s}} \longrightarrow \mathscr{O}_{Z} \longrightarrow 0
$$

где $\mathscr{J}$ - пучок идеалов $Z$ в $Y_{s}$. Отсюда получаем

$$
1-p_{a}\left(Y_{s}\right)=1-p_{a}(Z)+h^{0}(\mathscr{J})-h^{1}(\mathscr{J})
$$

или, эквивалентно, $p_{a}(Z)=1+h^{0}(\mathscr{J})-h^{1}(\mathscr{J})$. Так как $H^{0}\left(Y_{s}, \mathscr{O}_{Y_{s}}\right)=\mathbb{C}$, то $h^{0}(\mathscr{J})=0$. С другой стороны, по двойственности Серра

$$
h^{1}(\mathscr{J})=\operatorname{dim} \operatorname{Hom}\left(\mathscr{J}, \omega_{Y_{s}}\right)=\operatorname{dim} \operatorname{Hom}\left(\mathscr{J}, \mathscr{O}_{Y_{s}}\right)>0 .
$$

Следовательно, $p_{a}(Z) \leqslant 0$, что и доказывает предложение 3.6 .

СледСтвиЕ 3.8 (из предложения 3.6 (ii)). Существует вложение

$$
Y \hookrightarrow \mathbb{P}_{x_{0}, x_{1}, x_{2}, x_{3}}^{3} \times \mathbb{C}_{u, v}^{2}
$$

такое, что $Y$ задается двумя уравнениями, квадратичньми по $x_{0}, x_{1}, x_{2}, x_{3}$.

ОКОНЧАНИЕ ДОКАЗАТЕЛЬСТВА ТЕОРЕМЫ 3.1. Так как группа $\mathbb{Z}_{2}$ переводит дивизор $B$ в себя, то действие $\mathbb{Z}_{2}$ продолжается на $\mathbb{P}^{3} \times \mathbb{C}^{2}$, причем действие на втором сомножителе тривиально. Далее, пусть $P \in X$ - точка индекса 2, тогда $Q:=h^{-1}(P)$ - одна точка и $h:(Y, Q) \rightarrow(X, P)$ - каноническое накрытие. Согласно теоремам 1.1 и 1.2 действие $\mathbb{Z}_{2}$ в касательном пространстве Зарисского $T_{Q, Y} \subset T_{Q, \mathbb{P}^{3} \times \mathbb{C}^{2}}$ имеет трехмерное антиинвариантное подпространство. Отсюда линейными заменами координат можно добиться того, что действие $\mathbb{Z}_{2}$ на $\mathbb{P}_{x_{0}, x_{1}, x_{2}, x_{3}}^{3} \times \mathbb{C}_{u, v}^{2}$ будет как в (11). Множество неподвижных точек в $\mathbb{P}^{3} \times \mathbb{C}^{2}-$ это дивизор $\left\{x_{3}=0\right\}$, высекающий $B$ на $Y$, и поверхность $\left\{x_{0}=x_{1}=x_{2}=0\right\}$, высекаюшая изолированную точку $Q=\left\{x_{0}=x_{1}=x_{2}=u=v=0\right\}$ на $Y$ (по крайней мере локально вблизи слоя $Y_{o}$ ). Это доказьвает утверждение (iv) теоремы 3.1.

Следовательно, $P=h(Q)$ - единственная точка индекса 2 на $X$. Далее, легко видеть из следствия 3.8, что многообразие $Y$ может быть задано как базисное множество пучка квадрик от $x_{0}, \ldots, x_{3}$ с коэффициентами из $\mathbb{C}\{u, v\}$. Выберем две $\mathbb{Z}_{2}$-инвариантные квадрики из этого пучка. Получим уравнения для $Y$

$$
\left\{\begin{array}{l}
q_{1}\left(x_{0}, x_{1}, x_{2}\right)=\psi_{1}\left(x_{0}, x_{1}, x_{2}, x_{3} ; u, v\right) \\
q_{2}\left(x_{0}, x_{1}, x_{2}\right)=\psi_{2}\left(x_{0}, x_{1}, x_{2}, x_{3} ; u, v\right)
\end{array}\right.
$$


где $q_{1}, q_{2}-$ квадратичные функции, а функции $\psi_{1}, \psi_{2}-$ квадратичны по $x_{0}, x_{1}, x_{2}, x_{3}$ и $\psi_{i}\left(x_{0}, x_{1}, x_{2}, x_{3} ; 0,0\right)=0, i=1,2$. Отсюда слой $Y_{o}$ имеет вид $q_{1}=q_{2}=0$ и, следовательно, он является объединением прямых, проходящих через $Q$. Таким образом, утверждения (v) и (i) теоремы 3.1 доказаны. Наконец, уравнения $q_{1}\left(x_{0}, x_{1}, x_{2}\right)=q_{2}\left(x_{0}, x_{1}, x_{2}\right)=0$ задают пучок квадрик на $\mathbb{P}^{2}$. Проективным преобразованием мы можем привести его к одной из форм в (vi) теоремы 3.1. Теорема доказана.

Примеры. Ниже мы рассмотрим конкретные примеры расслоений Мори на коники индекса 2. В частности, мы покажем, что все случаи из утверждения (vi) теоремы 3.1 реализуются. Будут использованы обозначения п. 3.4 и теоремы 3.1 , а также, как и в [13], обозначения $c A / 2, c A x / 2, c D / 2, c E / 2$ для терминальных особенностей индекса два.

ПримеР 3.9. Пусть $Y \subset \mathbb{P}_{x_{0}, x_{1}, x_{2}, x_{3}}^{3} \times \mathbb{C}_{u, v}^{2}$ задается уравнениями

$$
\left\{\begin{array}{l}
x_{0} x_{1}=\left(a u+b u^{2}+c u v\right) x_{3}^{2} \\
\left(x_{0}+x_{1}+x_{2}\right) x_{2}=v x_{3}^{2}
\end{array}\right.
$$

где $a, b, c \in \mathbb{C}$ - константы. Легко проверить, что $Y$ неособо вблизи центрального слоя $g^{-1}(0)$, если $a \neq 0$, и имеет изолированную гиперповерхностную особенность в точке $Q=\left\{u=v=x_{0}=x_{1}=x_{2}=0\right\}$, если $a=0, c \neq 0, b \neq 0$ такую, что ранг квадратичной части равен 3 . Тогда фактор $X:=Y / \mathbb{Z}_{2} \rightarrow \mathbb{C}^{2}$ является расслоением Мори на коники, таким как в (a), (vi) теоремы 3.1, с единственной особой точкой $P$ типа $c A / 2$. Если $a \neq 0, P$ будет типа $\frac{1}{2}(1,1,1)$.

ПРИмеР 3.10. Пусть $Y \subset \mathbb{P}_{x_{0}, x_{1}, x_{2}, x_{3}}^{3} \times \mathbb{C}_{u, v}^{2}$ задается уравнениями

$$
\left\{\begin{array}{l}
x_{0} x_{1}=u\left(x_{0}^{2}+x_{1}^{2}+x_{2}^{2}-x_{3}^{2}\right)+a v x_{3}^{2}, \\
\left(x_{0}+x_{1}\right) x_{2}=v x_{3}^{2}+b u x_{0}^{2},
\end{array} \quad a, b \in \mathbb{C} .\right.
$$

Тогда фактор $X:=Y / \mathbb{Z}_{2} \rightarrow \mathbb{C}^{2}$ является расслоением Мори на коники как в (b), (vi) теоремы 3.1. $X$ содержит единственную негоренштейнову точку типа $\frac{1}{2}(1,1,1)$. Если $a=b=0$, то $X$ имеет также обыкновенную двойную особую точку на кратной компоненте $Y_{o}$.

ПРимеР 3.11. Пусть $Y \subset \mathbb{P}_{x_{0}, x_{1}, x_{2}, x_{3}}^{3} \times \mathbb{C}_{u, v}^{2}$ задается уравнениями

$$
\left\{\begin{array}{l}
x_{0} x_{1}-x_{2}^{2}=u x_{3}^{2} \\
x_{0} x_{2}=u x_{1}^{2}+v\left(x_{2}^{2}+x_{3}^{2}\right)
\end{array}\right.
$$

Тогда фактор $X:=Y / \mathbb{Z}_{2} \rightarrow \mathbb{C}^{2}-$ расслоение Мори на коники с единственной особой точкой типа $\frac{1}{2}(1,1,1)$, такое как в (c), (vi) теоремы 3.1 .

Следующий пример показывает, что все типы терминальных особенностей индекса 2 могут быть реализованы на расслоениях Мори на коники, таких как в (d), (vi) теоремы 3.1 . 
ПримеР 3.12. Пусть $Y \subset \mathbb{P}_{x_{0}, x_{1}, x_{2}, x_{3}}^{3} \times \mathbb{C}_{u, v}^{2}$ задается уравнениями

$$
\left\{\begin{array}{l}
x_{0} x_{1}=u x_{3}^{2}, \\
x_{2}^{2}=u\left(x_{0}^{2}+x_{1}^{2}\right)+\left(a v+b v^{2}+c v^{3}+d u v+e u^{2} v\right) x_{3}^{2},
\end{array}\right.
$$

где $a, b, c, d, e \in \mathbb{C}$ - константы. Если по крайней мере одна из констант $a, b, c$ отлична от нуля, то $Y$ имеет вблизи центрального слоя $Y_{0}$ изолированную особенность в $Q=\left\{x_{0}=x_{1}=x_{2}=u=v=0\right\}$ (или неособо). Как и выше, фактор $X:=Y / \mathbb{Z}_{2} \rightarrow \mathbb{C}^{2}-$ расслоение Мори на коники такое как в $(\mathrm{d}),(\mathrm{vi})$ теоремы $3.1 \mathrm{c}$ единственной особой точкой $P:=C_{1} \cap C_{2}$. Мы имеем следующие возможности:

1) $a \neq 0$, тогда $Y$ неособо, таким образом, $P \in X$ - типа $\frac{1}{2}(1,1,1)$,

2) $a=0, b \neq 0$, тогда $P \in X-$ типа $c A x / 2$,

3) $a=b=e=0, c \neq 0, d \neq 0$, тогда $P \in X-$ типа $c D / 2$,

4) $a=b=d=0, c \neq 0, e \neq 0$, тогда $P \in X-$ типа $c E / 2$.

ПримеР 3.13. Пусть $Y \subset \mathbb{P}_{x_{0}, x_{1}, x_{2}, x_{3}}^{3} \times \mathbb{C}_{u, v}^{2}$ задается уравнениями

$$
\left\{\begin{array}{l}
x_{0} x_{1}-x_{2}^{2}=u x_{3}^{2} \\
x_{0}^{2}=u x_{2}^{2}+v\left(x_{2}^{2}+x_{3}^{2}\right)
\end{array}\right.
$$

Тогда фактор $X:=Y / \mathbb{Z}_{2} \rightarrow \mathbb{C}^{2}$ является расслоением Мори на коники как в (е), (vi) теоремы 3.1.

Отметим, что для любого расслоения Мори на коники $f:(X, C) \rightarrow(S, o)$ с приводимым центральным слоем и любой компоненты $C^{\prime} \subset C$ росток $\left(X, C^{\prime}\right)$ является экстремальной окрестностью (не обязательно изолированной) в смысле Мори [8]. Тогда $X$ может иметь на $C^{\prime}$ не более одной горенштейновой особой точки (см. $[8,6.2])$. Следующий пример показывает, что это правило нарушается, если слой $C$ неприводим.

ПримеР 3.14. Пусть $Y \subset \mathbb{P}_{x_{0}, x_{1}, x_{2}, x_{3}}^{3} \times \mathbb{C}_{u, v}^{2}$ задается уравнениями

$$
\left\{\begin{array}{l}
x_{0}^{2}=u x_{2}^{2}+v x_{3}^{2} \\
x_{1}^{2}=u x_{3}^{2}+v x_{2}^{2}
\end{array}\right.
$$

Тогда фактор $X:=Y / \mathbb{Z}_{2} \rightarrow \mathbb{C}^{2}$ является расслоением Мори на коники как в (f), (vi) теоремы 3.1 . В этом случае $X$ содержит одну точку типа $\frac{1}{2}(1,1,1)$ и две обькновенные двойные точки.

В заключение мы исследуем расслоения Мори на коники индекса 2 с особой базой.

Teорема 3.15. Пусть $f:(X, C) \rightarrow(S, o)$ - расслоение Мори на коники индекса 2. Предположим, что точка $(S$, o) особа. Тогда $(S, o)$ - дювалевская точка типа $A_{1}$ u $f$ такое, как в примере 2.3 или как в примере 2.1 с $n=2$. 
ДокАЗАТЕЛЬСТво. Мы будем использовать конструкцию 1.9. Так как $X$ содержит только точки индексов $\leqslant 2$, то по следствию $1.11(S, o)$ - особенность типа $A_{1}$. Мы утверждаем, что $f^{\prime}:\left(X^{\prime}, C^{\prime}\right) \rightarrow\left(\mathbb{C}^{2}, 0\right)$ - (обычное) расслоение на коники. По предложению 1.6 достаточно доказать, что $X^{\prime}$ горенштейново. Предположим противное. Отметим, что $X^{\prime}$ содержит только точки индексов $\leqslant 2$, так как $X^{\prime} \rightarrow X$ неразветвлено в коразмерности 1 . По теореме $3.1 X^{\prime}$ содержит единственную точку, обозначим ее через $Q$, индекса 2 . Тогда $Q$ - неподвижная точка относительно действия $\mathbb{Z}_{2}$. Но тогда точка $P:=h(Q)$ имеет индекс 4 , противоречие. Следовательно, $f^{\prime}$ - расслоение на коники и по теореме $2.4 f:(X, C) \rightarrow(X, C)$ такое, как в примере 2.1 или 2.3. Это доказывает теорему.

\section{§4. Классификация при условии существования хорошего дивизора}

В этом параграфе мы будем изучать расслоения Мори на коники при предположении сушествования хорошего элемента в $\left|-K_{X}\right|$. Отметим, что во всех известных примерах такой элемент существует.

Пример 4.1. Пусть расслоение Мори на коники $f:(X, C) \rightarrow(S, o)$ такое, как в примере 2.2. Рассмотрим открытое подмножество $U_{2}=\left\{x_{2} \neq 0\right\} \subset X$. Локальньми координатами в $U_{2}$ являются $\left(t_{0}=x_{0} / x_{2}, t_{1}=x_{1} / x_{2}, v\right)$. Рассмотрим также рациональную дифференциальную форму $\sigma=\left(1 / t_{0}\right)\left(d t_{0} \wedge d t_{1} \wedge d v\right)$ на $U_{2}$. Тогда легко видеть, что $\sigma$ может быть продолжена на $X^{\prime}$ в окрестности $C^{\prime}$. Так как форма $\sigma \mathbb{Z}_{n}$-инвариантна, $\sigma^{-1}$ определяет элемент $F \in\left|-K_{X}\right|$, образ дивизора $\left\{x_{0}=0\right\}$. Легко также проверить, что $F$ содержит центральный слой $C=\left(f^{-1}(o)\right)_{\text {red }}$ и имеет две особые точки типа $A_{n-1}$.

Аналогично, можно проверить, что в примере 2.1 общий элемент $F \in\left|-K_{X}\right|$ не содержит $C$ и состоит из двух связных компонент. Этот дивизор имеет особую точку типа $A_{n-1}$ на каждой компоненте.

Следуюшая теорема немного улучшает результаты [1].

Tеорема 4.2. Пусть $f:(X, C) \rightarrow(S, o)$ - расслоение Мори на коники. Предположим, что гипотеза 0.2 для $f$ верна. Тогда выполнено одно из следующих условий:

(i) точка $(S$, о) неособа;

(ii) точка $(S, o)$ - дювалевская типа $A_{1}$;

(iii) точка $(S, o)$ - дювалевская типа $A_{3}$, в этом случае кривая $C$ неприводима $(X, C)$ имеет ииклическую факторособенность $P$ индекса 8 и не имеет других особых точек. Более того, $f:(X, C) \rightarrow(S$, о) является фактором расслоения Мори на коники из (a), (vi) теоремы 3.1 с неособъцм $Y$ по группе $\mathbb{Z}_{4}$, действующей на базе свободно в коразмерности 1 (см. также [4]).

(iv) $f:(X, C) \rightarrow(S$, o) такое, как в примере 2.1 или примере 2.2. В частности, $(S, o)$ имеет тип $A_{n-1}$ в әтом случае.

ДокАЗАТЕЛЬСтво. Предположим, что точка $(S, o)$ особа и она не имеет типа $A_{1}$. Мы будем использовать обозначения конструкции 1.9. Если $X^{\prime}$ имеет индекс 1 , то по предложению $1.6 f^{\prime}$ - расслоение на коники и по теореме $2.4 f$-из 
примера 2.1 или из примера 2.2. Таким образом, мы предположим, что $X^{\prime}$ индекса $>1$ и покажем в этом случае, что $f:(X, C) \rightarrow(S, o)$ такое, как в (iii). Пусть $F \in\left|-K_{X}\right|$ - общий дивизор и $F^{\prime}:=h^{-1}(F)$. По нашему предположению $F$ имеет только дювалевские особенности и так как накрытие $F^{\prime} \rightarrow F$ неразветвлено в коразмерности $1, F^{\prime}$ также имеет только дювалевские особенности (ср. с леммой 1.4). Так как $\left(-K_{X} \cdot X_{s}\right)=2$, где $X_{s}$ - общий слой $f$, ограничение $\left.f\right|_{F}: F \rightarrow S$ в общей точке конечно степени 2. Имеются следующие случаи $4.3,4.4$ и 4.6 .

4.3. Множество $F^{\prime} \cap C^{\prime}$ не связно. Как и вьше, так как $F^{\prime} \in\left|-K_{X^{\prime}}\right|$, мы видим, что морфизм $\left.f\right|_{F^{\prime}}: F^{\prime} \rightarrow S^{\prime}$ в общей точке конечен степени 2 и $F^{\prime}$ имеет две компоненты связности $F_{1}^{\prime}$ и $F_{2}^{\prime}$. Пусть

$$
f^{\prime}: F^{\prime} \stackrel{f_{1}^{\prime}}{\longrightarrow} D^{\prime} \stackrel{f_{2}^{\prime}}{\longrightarrow} S^{\prime}
$$

- факторизация Штейна. Тогда морфизм $f_{1}^{\prime}: F^{\prime} \rightarrow D^{\prime}$ бимероморфен, а $f_{2}^{\prime}: D^{\prime} \rightarrow S^{\prime}$ - конечный степени 2. В нашем случае $D^{\prime}$ имеет ровно две неприводимые компоненты $D_{1}^{\prime}$ и $D_{2}^{\prime}$. Следовательно, мы имеем $D_{1}^{\prime} \simeq D_{2}^{\prime} \simeq S^{\prime}$ и дивизор $D^{\prime}$ неособ (так как неособа поверхность $S^{\prime}$ ). С другой стороны, по формуле присоединения $K_{F^{\prime}}=0$. Следовательно, морфизм $f_{1}^{\prime}$ крепантный (т.е. $\left.K_{F^{\prime}}=f_{1}{ }^{*} K_{D^{\prime}}\right)$. Это означает, что $F^{\prime}$ неособ и $f_{1}^{\prime}=\mathrm{id}$. По лемме $1.4 X^{\prime}$ не содержит точек индекса $>1$, противоречие с нашшми предположениями в начале доказательства.

4.4. $F^{\prime} \cap C^{\prime}$ - одна точка $Q$. Тогда $F \cap C=\{h(Q)\}$ - также одна точка $P$. Следовательно, морфизм $\left.f\right|_{F}: F \rightarrow S$ конечен степени 2 и $P$ - единственная точка индекса $>1$. Таким образом, $(F, P) \rightarrow(S, o)$ - двойное накрытие изолированных особенностей. Отсюда $(S, o)$ - фактор дювалевской особенности $(F, P)$ по некоторой инволюции $\tau$.

Действия инволюций на дювалевских особенностях были классифицированы Катанезе [26]. Напомним, что $(S, o)$ имеет топологический индекс $n \geqslant 3$ (иначе мы имеем случаи (i) или (ii) нашей теоремы). Учитывая что $(S, o)$ - циклический фактор, из списка в [26] мы получим следующее

ПРЕДЛОЖЕНИЕ 4.5 [26]. Пусть $(F, P)$ - росток дювалевской особенности и $\tau$ - аналитическая инволющия, действующая на $(F, P)$. Предположсим, что фактор $(S, o):=(F, P) / \tau$ является чиклической факторособенностью типа $\frac{1}{n}(a, b)$ с $n \geqslant 3(u(a, n)=(b, n)=1)$. Тогда имеются следуюшие возможсности $\partial \bumpeq я(F, P) \rightarrow(S, o):$

$$
\begin{array}{rlrl}
E_{6} & \stackrel{2: 1}{\longrightarrow} A_{2}, & n=3, \\
A_{2 n-1} & \stackrel{2: 1}{\longrightarrow} A_{n-1}, & n \geqslant 3, \\
A_{2 k} \stackrel{2: 1}{\longrightarrow} \frac{1}{2 k+1}(k, 2 k-1), & n=2 k+1, k \geqslant 1, \\
A_{k} \stackrel{2: 1}{\longrightarrow} A_{2 k+1}, & n=2 k+1, k \geqslant 1, \\
A_{2 k+1} \stackrel{2: 1}{\longrightarrow} \frac{1}{4 k+4}(2 k+1,2 k+1), & n=4 k+4 k \geqslant 0 .
\end{array}
$$


Продолжим рассмотрение случая 4.4. Ограничение $\left.h\right|_{F^{\prime}}:\left(F^{\prime}, Q\right) \rightarrow(F, P)$ неразветвлено вне $P$ и имеет степень $n$. Следовательно, точка $\left(F^{\prime}, Q\right)$ - дювалевская. Легко видеть (см., например, $[12,4.10])$, что $(F, P)$ не имеет таких накрытий в случаях $(16),(17)$. Особенность $(F, P)$ из (15) допускает только накрытие неособой точкой $\left(F^{\prime}, P^{\prime}\right)$ степени $n=2 k+1$. Но тогда $\left(X^{\prime}, Q\right)$ - неособая точка по лемме 1.4, противоречие с нашими предположениями в начале доказательства.

Пусть $m$ - индекс $(X, P), \pi:\left(X^{\#}, P^{\#}\right) \rightarrow(X, P)$ - каноническое накрытие и положим $F^{\#}:=\pi^{\#-1} F$. Как выше, имеем неразветвленное в коразмерности 1 $\mathbb{Z}_{m}$-накрытие $\pi:\left(F^{\#}, P^{\#}\right) \rightarrow(F, P)$ дювалевских особенностей. Так как $\pi$ пропускается через $\left(X^{\prime}, Q\right)$, мы имеем $m \geqslant n$.

В случае $(13)(F, P)=E_{6}$ по $[12,4.10]$ и допускает только циклическое накрытие типа $D_{4} \stackrel{3: 1}{\longrightarrow} E_{6}$. Тогда $n=m=3$. Следовательно, $\left(X^{\#}, P^{\#}\right) \simeq\left(X^{\prime}, Q\right)$ имеет индекс 1 , противоречие.

Наконец, рассмотрим случай (14). Так как $\left(X^{\prime}, Q\right)$ имеет индекс $>1$, мы имеем $m>n \geqslant 3$ и по $[12,4.10]$ накрытие $\left(F^{\#}, P^{\#}\right) \stackrel{m: 1}{\longrightarrow}(F, P)$ типа (неособая точка) $\stackrel{2 n: 1}{\longrightarrow} A_{2 k+1}, m=2 n$. Тогда индекс $\left(X^{\prime}, Q\right)$ равен $m / n=2$, точка $\left(X^{\#}, P^{\#}\right)$ неособа, и, следовательно, $\left(X^{\prime}, Q\right)$ - циклическая факторособенность типа $\frac{1}{2}(1,1,1)$. В частности, $f$ - такое, как в теореме 3.1. Пусть $X_{o^{\prime}}^{\prime}=f^{\prime-1}\left(o^{\prime}\right)$ - схемно-теоретический слой $f^{\prime}$ над $o^{\prime}$. Далее $Q$ - единственная неподвижная точка на $C^{\prime}$ при действии $\mathbb{Z}_{n}$. Следовательно, $\mathbb{Z}_{n}$ переставляет компоненты $\left\{C_{i}^{\prime}\right\}$ кривой $C^{\prime}$. В частности, число компонент $\geqslant n \geqslant 3$ и кратности компонент в $X_{o^{\prime}}^{\prime}$ равны между собой. Отсюда для $f^{\prime}:\left(X^{\prime}, C^{\prime}\right) \rightarrow\left(S^{\prime}, o^{\prime}\right)$ мы получаем только одну возможность (a), (vi) теоремы 3.1. Поэтому $X_{o}^{\prime}$ имеет ровно четыре компоненты, $n=4$ и слой $X_{o}^{\prime}$ приведен. Тогда $X^{\prime}$ неособо вне $Q$. Мы получаем случай (iii) нашей теоремы.

4.6. Множество $F^{\prime} \cap C^{\prime}$ одномерно и связно. Тогда такое же $F \cap C$. Пусть $L:=F^{\prime} \cap C^{\prime}$ (с приведенной структурой). На поверхности $F^{\prime}=h^{-1}(F)$ мы имеем по формуле присоединения, что кривая $L$ горенштейнова и $\omega_{L}=\mathscr{O}_{L}(L)$ (так как $\omega_{F^{\prime}}=\mathscr{O}_{F^{\prime}}$ ). Так как $L$ стягивается морфизмом $f^{\prime}$, дуализируюший пучок $\omega_{L}$ антиобилен. Следовательно, $L$ изоморфна приведенной конике в $\mathbb{P}^{2}$ (см., например, [27]), т.е. $L \simeq \mathbb{P}^{1}$ или $L$ - пара пересекающихся прямых.

Пусть

$$
f_{F}: F \stackrel{f_{1}}{\longrightarrow} D \stackrel{f_{2}}{\longrightarrow} S
$$

- факторизация Штейна. Тогда морфизм $f_{1}: F \rightarrow D$ бимероморфен, а $f_{2}: D \rightarrow S$ конечен степени 2. По формуле присоединения $K_{F}=0$. Следовательно, морфизм $f_{1}$ крепантен (т.е. $\left.K_{F}=f_{1}^{*} K_{D}\right)$ и $D$ имеет только дювалевские особенности.

Существует общее минимальное разрешение $\sigma: \widetilde{F} \rightarrow F \rightarrow D$. В нашем случае $f_{2}^{-1}(o)$ состоит из единственной точки $R=f_{1}(F \cap C)$ (и это - единственная особая точка на $D)$. Пусть $\Gamma=\Gamma(\widetilde{F} / D)$ - двойственный граф для $\sigma$. Обозначим вершины, соответствующие компонентам $h(L)$ (соответственно, $f_{2}$-исключительным дивизорам), через • (соответственно, о). Тогда белые вершины образуют связные подграфы, соответствуюшие особым точкам $(F, C)$, а черные вершины соответствуют компонентам $C$, которые содержатся в $F$. Так как $f_{1}^{-1}(R)=L / \mathbb{Z}_{2}$, то $Г$ имеет не более двух черных вершин. Для $f_{2}:(D, R) \rightarrow(S, o)$ мы имеем те же возможности, что и для $(F, P) \rightarrow(S, o)$ в предложении 4.5. Рассмотрим эти подслучаи. 
Подслучай $(13) .(D, R)=E_{6},(S, o)=A_{2}, n=3$. Группа $\mathbb{Z}_{3}$ естественно действует на кривой $L:=F^{\prime} \cap C^{\prime}$. Так как $L-$ коника, $\mathbb{Z}_{3}$ не может переставлять ее компоненты. Следовательно, $\mathbb{Z}_{3}$ имеет две или три неподвижные точки $Q_{i}$ на $L$. Тогда существуют по крайней мере две точки $P_{1}, P_{2} \in X$ индексов $\geqslant 3$. Эти точки на $F$ имеют по лемме 1.5 топологические индексы $\geqslant 3$. С другой стороны, графф минимального разрешения $\left(F, P_{i}\right)$ должен быть белым подграфом в $\Gamma\left(\simeq E_{6}\right)$. Следовательно, каждая $\left(F, P_{i}\right)$ имеет тип $A_{2}$ и существует только две неподвижных точки на $L$. Это возможно только, если кривая $L$ неприводима и такова же $h(L)=F \cap C$. Таким образом, мы имеем только одну возможность для $\Gamma$

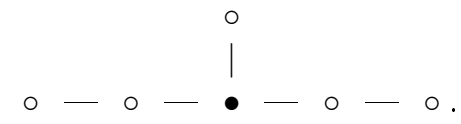

Следовательно, $F$ содержит ровно две особые точки $P_{1}, P_{2}$ и они имеют тип $A_{2}$. Многообразие $X$ имеет в этих точках индекс 3 . Так как $h^{-1}\left(P_{i}\right)=Q_{i}$ по лемме 1.5 , $X^{\prime}$ горенштейново. Мы получим противоречие с нашими предположениями.

Подслучай (14). $(D, R)=A_{2 n-1},(S, o)=A_{n-1}$. Если кривая $L$ неприводима или $\mathbb{Z}_{n}$ не переставляет ее компонент, то, как и вьше, $F$ содержит по крайней мере две точки с топологическими индексами $\geqslant n$. Тогда $\Gamma\left(=A_{2 n-1}\right)$ будет

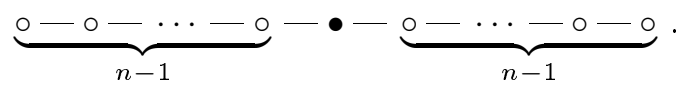

Следовательно, $F$ содержит ровно две особые точки и они имеют тип $A_{n-1}$. По лемме $1.5 X^{\prime}$ горенштейново, противоречие.

Следовательно, кривая $L$ приводима и $\mathbb{Z}_{n}$ переставляет ее компоненты. Тогда $h(L)=F \cap C$ неприводима и поэтому Г имеет только одну черную вершину. Далее, $\mathbb{Z}_{n}$-неподвижная точка $Q_{1}$ на $X^{\prime}$ дает нам точку $P_{1} \in F$ топологического индекса $n k, k \in \mathbb{N}$ (см. лемму 1.5$)$. Она соответствует белому подграфу в $Г$ типа $A_{n k-1}$. Таким образом, $k=1$ и $Г$ снова имеет вид (18). Отсюда $\left(F, P_{1}\right)$ - типа $A_{n-1}$. В частности, по лемме $1.4 Q_{1}$ неособа. Второй белый подграф̆ в $\Gamma$ также соответствует особой точке $P_{2} \in F$ типа $A_{n-1}$. Тогда индекс $\left(X, P_{2}\right)$ делит $n$. Так как $\mathbb{Z}_{n}$ переставляет две компоненты $L, n=2 l$ четно и сушествует три $\mathbb{Z}_{l}$-неподвижные точки на $L: Q_{1}, Q_{2}$ и $Q_{3}$, где $h\left(Q_{2}\right)=h\left(Q_{3}\right)=P_{2}$. Следовательно, индекс $P_{2}$ делится на $l$. По нашему предположению $\left(X^{\prime}, C^{\prime}\right)$ негоренштейново, следовательно, $\left(X^{\prime}, Q_{2}\right),\left(X^{\prime}, Q_{3}\right)$ имеют (один и тот же) индекс $m_{0}>1$ и $\left(X^{\prime}, C^{\prime}\right)$ не содержит других точек индекса $>1$. Но тогда индекс $(X, R)$ равен $m_{0} l=m_{0} n / 2$ и делит $n$. Следовательно, $m_{0}=2$, это означает, что $f^{\prime}:\left(X^{\prime}, C^{\prime}\right) \rightarrow\left(S^{\prime}, o^{\prime}\right)$ - расслоение Мори на коники индекса 2 , которое содержит две негоренштейновы точки $Q_{2}, Q_{3}$, противоречие с теоремой 3.1 .

Наконец, случаи (15), (16) и (17) невозможны, как в п. 4.4.

\section{§5. Пример расслоения Мори на коники индекса 3}

В заключение мы построим пример расслоения Мори на коники индекса 3 с неособой базой (и, следовательно, отличное от примеров 2.1 и 2.2 ). 
Рассмотрим подмногообразие $V \subset \mathbb{P}_{x_{0}, x_{1}, x_{2}, x_{3}}^{3} \times \mathbb{C}_{u, v}^{2}$, заданное двумя уравнениями

$$
\left\{\begin{array}{l}
a_{1} x_{1}^{3}+a_{2} x_{2}^{3}+a_{3} x_{1}^{2} x_{2}+a_{4} x_{2}^{2} x_{1}+a_{5} x_{3}^{2} x_{1}+a_{6} x_{3}^{2} x_{2}=u x_{0}^{3}, \\
b_{1} x_{1}^{3}+b_{2} x_{2}^{3}+b_{3} x_{1}^{2} x_{2}+b_{4} x_{2}^{2} x_{1}+b_{5} x_{3}^{2} x_{1}+b_{6} x_{3}^{2} x_{2}=v x_{0}^{3},
\end{array}\right.
$$

где $a_{i}, b_{i} \in \mathbb{C}$ - достаточно общие константы. Тогда $V$ неособо и проекция на второй множитель $q: V \rightarrow \mathbb{C}^{2}$ задает расслоение на кривые степени 9 в $\mathbb{P}_{x_{0}, x_{1}, x_{2}, x_{3}}^{3} \times$ $\mathbb{C}_{u, v}^{2}$. Центральный слой $q^{-1}(0)$ имеет ровно девять компонент $\Gamma_{0}, \ldots, \Gamma_{8}$, где $\Gamma_{0}:=\left\{u=v=x_{1}=x_{2}=0\right\}$. Определим действие $\mathbb{Z}_{6}$ на $V$ следующим обра30M

$$
\left(x_{0}, x_{1}, x_{2}, x_{3} ; u, v\right) \rightarrow\left(\varepsilon x_{0}, x_{1},-x_{2}, x_{3} ; u, v\right), \quad \varepsilon=\exp (2 \pi i / 3) .
$$

Сначала рассмотрим фактор $p: V \rightarrow Y:=V / \mathbb{Z}_{3}$ и естественную проекцию $g: Y \rightarrow$ $\mathbb{C}^{2}$. Множество $\mathbb{Z}_{3}$-неподвижных точек - это дивизор $R:=\left\{x_{0}=0\right\} \cap V$ и изолированная точка $O:=\left\{x_{1}=x_{2}=x_{3}=u=v=0\right\}$. Локальные координаты на $V$ в окрестности $O$ будут $\left(x_{1} / x_{0}, x_{2} / x_{0}, x_{3} / x_{0}\right)$ с $\mathbb{Z}_{3}$-весами

$$
\operatorname{wt}\left(x_{1} / x_{0}\right)=\operatorname{wt}\left(x_{2} / x_{0}\right)=\operatorname{wt}\left(x_{3} / x_{0}\right)=-1 .
$$

Следовательно, особое множество $Y$ состоит из единственной точки типа $\frac{1}{3}(1,1,1)$ (обозначим ее через $Q$ ), она является канонической горенштейновой. Далее по формуле Гурвица имеем

$$
K_{V}=p^{*} K_{Y}+2 R, \quad p^{*} K_{Y}=K_{V}-2 R=0 .
$$

Поэтому канонический дивизор $Y$ тривиален и $g: Y \rightarrow \mathbb{C}^{2}-$ расслоение на эллиптические кривые. Так как $\mathbb{Z}_{3}$ не переставляет кривые $\Gamma_{0}, \ldots, \Gamma_{8}$, то центральный слой $g^{-1}(0)$ состоит из девяти компонент $L_{0}, L_{1}, \ldots, L_{8}$, которые являются образами соответствующих компонент $\Gamma_{0}, \ldots, \Gamma_{8}$ слоя $q^{-1}(0)$.

Теперь рассмотрим фактор $h: Y \rightarrow X:=Y / \mathbb{Z}_{2}$ и естественньй морфизм $f: X \rightarrow \mathbb{C}^{2}$. Компонента $L_{0} \mathbb{Z}_{2}$-инвариантна, а $\mathbb{Z}_{2}$ переставляет $L_{i}, i=1, \ldots, 8$, нетривиально. Следовательно, $f^{-1}(0)=g^{-1}(0) / \mathbb{Z}_{2}$ имеет ровно пять компонент. Обозначим их через $C_{0}, \ldots, C_{4}$. Для того чтобы доказать, что $f: X \rightarrow \mathbb{C}^{2}$ - pacслоение Мори на коники, мы должны исследовать особое множество многообразия $X$. Множество $\mathbb{Z}_{2}$-неподвижных точек на $V$ составляет неприводимый дивизор $D:=\left\{x_{2}=0\right\} \cap V$. Поэтому множество $\mathbb{Z}_{2}$-неподвижных точек на $Y$ составляет в точности один неприводимьй дивизор Вейля $F:=p(D)$ такой, что $3 F$ является дивизором Картье. Более того, $D$ пересекает компоненты $\Gamma_{0}, \ldots, \Gamma_{8}$ трансверсально.

Следуюшая лемма является легким упражнением.

Лемма 5.1. Пусть $(Y, Q)$ - ииклическая факторособенность типа $\frac{1}{3}(1,1,1)$ с действием группь $\mathbb{Z}_{2}$. Предположсим, что множсество неподвижсных точек является дивизором Вейля $F$ таким, что $3 F \sim 0$. Тогда $(Y, Q) / \mathbb{Z}_{2}$ терминальная точка типа $\frac{1}{3}(1,1,-1)$.

Наконец, по формуле Гурвица $0=K_{Y}=h^{*}\left(K_{X}\right)+F$. Так как $\left(L_{i} \cdot F\right)>0$, мы имеем $\left(h^{*}\left(-K_{X}\right) \cdot L_{i}\right)=\left(F \cdot L_{i}\right)>0, i=0, \ldots, 8$. Поэтому $\left(-K_{X} \cdot C_{i}\right)>0$, $i=0, \ldots, 4$, т.е. дивизор $-K_{X}$ относительно $f$-обилен (по крайней мере вблизи $\left.f^{-1}(0)\right)$. 
Вывод. Мы построили расслоение Мори на коники $f:(X, C) \rightarrow\left(\mathbb{C}^{2}, 0\right)$ такое, что его центральный слой $C$ имеет ровно пять компонент и (единственная) особая точка $P$ на $X$ является циклической факторособенностью типа $\frac{1}{3}(1,1,-1)$. Все компоненты $C$ проходят через $P$ и не пересекаются вне. Отметим, что накрытие $h: Y \rightarrow X-$ это в точности трюк с двойньм накрытием Каваматы [28].

\section{Список литературы}

1. Прохоров Ю. Г. К проблеме общего слона для трехмерных $\mathbb{Q}$-Фано расслоений над поверхностью // Фундаментальная и прикладная математика 1995. Т. 1. № 1. С. 263-280.

2. Prokhorov $Y$ u. G. On $\mathbb{Q}$-Fano fiber spaces with two-dimensional base // Preprint MPI 95/33.

3. Prokhorov Yu. G. On extremal contractions from 3-folds to surfaces // Preprint, Warwick $84 / 1995$.

4. Prokhorov Yu. G. On extremal contractions from 3-folds to surfaces: the case of one non-Gorenstein point // Contemporary Math. AMS. 1997. V. 207. P. 119-141.

5. Исковских В. А. К проблеме рациональности для расслоений на коники // Матем. сб. 1991. Т. 182 . № 1. С. $114-121$.

6. Исковских В. А. О критерии рационалњности для расслоений на коники // Матем. сб. 1996. T. 187. № 7. C. $75-92$.

7. Шокуров В. В. Трехмерные логперестройки // Изв. АН СССР. Сер. матем. 1992. Т. 56. № 1. C. 105-201; 1993. Т. 57. №6. C. 141-175.

8. Mori S. Flip theorem and the existence of minimal models for 3-folds // J. Amer. Math. Soc. 1988. V. 1. № 1. P. 117-253.

9. Reid M. Minimal models of canonical 3-folds. Algebraic Varieties and Analytic Varieties // Adv. Stud. Pure Math. 1983. V. 1. P. 131-180.

10. Данилов В. И. Бирациональная геометрия торических многообразий // Изв. АН СССР. Сер. матем. 1982. Т. 46. № 5. С. 971-982.

11. Mori S. On 3-dimensional terminal singularities // Nagoya Math. J. 1985. V. 98. P. 43-66.

12. Reid $M$. Young persons guide to canonical singularities. Algebraic Geometry, Bowdoin, 1985 // Proc. Symp. Pure Math. 1987. V. 46. P. 345-414.

13. Kollár J., Mori S. Classification of three-dimensional flips // J. Amer. Math. Soc. 1992. V. 5. № 3. P. 533-703.

14. Cutkosky S. Elementary contractions of Gorenstein 3-folds // Math. Ann. 1988. V. 280. P. 521-525.

15. Kollár J., Miyaoka Y., Mori S. Rationally connected varieties // J. Algebraic Geometry. 1992. V. 1. P. 429-448.

16. Ishii S. On Fano 3-folds with non-rational singularities and two-dimensional base // Abh. Math. Sem. Univ. Hamburg. 1994. V. 64. P. 249-277.

17. Клеменс $X$., Коллар Я., Мори C. Многомерная комплексная геометрия. М.: Мир, 1993.

18. Kollár J. Standard models of elliptic 3-folds // Preprint.

19. Cartan $H$. Quotient d'une space analytique par un groupe d'automorphismes // Algebraic Geometry and Topology. Princeton: Princeton Univ. Press, 1957. P. 90-102.

20. Beauville A. Variétés de Prym et jacobienne intermédiaires // Ann. Sci. Ecole Norm. Sup. (4). 1977. V. 10. P. 309-391.

21. Kawamata Y., Matsuda K., Matsuki K. Introduction to the minimal model program. Algebraic Geometry, Sendai, 1985 // Adv. Stud. Pure Math. 1987. V. 10. P. 283-360.

22. Kempf G. Cohomology and convexity. Toroidal Embeddings I // Lect. Notes in Math. V. 339, 1973. P. 41-52.

23. Matsumura H. Commutative Ring Theory. Cambridge: Cambridge Univ. Press, 1986.

24. Catanese F., Franciosi M., Hulek K., Reid M. Embeddings of curves and surfaces // Preprint alg-geom, 1986.

25. Hartshorne R. Residues and duality // Lect. Notes in Math. V. 20, 1966. 
26. Catanese F. Automorphisms of rational double points and moduli spaces of surfaces of general type // Compositio Math. 1987. V. 61. № 1. P. 81-102.

27. Lipman J. Rational singularities with applications to algebraic surfaces and unique factorization // Publ. Math. IHES. 1969. V. 36. P. 195-279.

28. Kawamata $Y$. Crepant blowing-ups of 3-dimensional canonical singularities and its application to degenerations of surfaces // Ann. Math. 1988. V. 127. P. 93-163.

Московский государственньй

Поступила в редакцию университет им. М.В. Ломоносова

E-mail: prokhoro@mech.math.msu.su prokhoro@nw.math.msu.su 